GASTRIC CANCER

\title{
Comparison of cyclooxygenase 2 expression in adenocarcinomas of the gastric cardia and distal oesophagus
}

\section{J Buskens, A Sivula, B P van Rees, C Haglund, G J A Offerhaus, J J B van Lanschot, A Ristimäki}

See end of article for authors' affiliations

Correspondence to: Dr C J'Buskens, Academic Medical Centre, Department of Surgery, Suite G4-130

Meibergdreef' 9, $1105 \mathrm{AZ}$ Amsterdam, the

Netherlands;

C.J.Buskens@amc.uva.nl

Accepted for publication 23 July 2003
Background: Adenocarcinomas of the gastric cardia and distal oesophagus are at present often considered as one clinical entity because of their comparable increasing incidence, prognosis, and optimal treatment options. However, it is still a matter of debate whether these malignancies have the same pathogenesis and genotype.

Aims: The aim of this study was to analyse expression of cyclooxygenase 2 (COX-2) in cardia carcinomas, and correlate this expression with clinicopathological parameters and survival. The results were compared with the prognostic value of COX-2 found for Barrett carcinomas.

Methods: Tumour sections of 134 consecutive patients undergoing potentially curative surgery for an adenocarcinoma of the gastric cardia and substantially invading the distal oesophagus were immunohistochemically stained using a COX-2 monoclonal antibody. Specimens were blindly scored based on intensity and extent of COX-2 immunopositivity.

Results: COX-2 expression was negative to weak in 59\% ("COX-2 low") and moderate to strong in $41 \%$ ("COX-2 high") of tumours. This was significantly lower than in Barrett carcinomas $(p<0.0001)$. COX-2 expression was not correlated with any clinicopathological parameter. A correlation between elevated COX-2 expression and reduced survival, as described for Barrett carcinomas, was not identified for cardiac carcinomas.

Conclusions: There is a difference in COX-2 expression with respect to intensity and prognostic significance between adenocarcinomas of the gastric cardia and distal oesophagus. This suggests a different pathogenesis and different genetic constitution of these two cancers. Based on these findings, the role of selective COX-2 inhibitors in the treatment of adenocarcinomas of the gastric cardia is less promising than in Barrett carcinomas.
G astric cancer is a highly aggressive disease. Worldwide, it ranks second after lung cancer among malignancy associated deaths. Gastric cancer can be subdivided into distal gastric malignancies and proximal cardia carcinomas. The incidence of distal gastric carcinoma has decreased from $32 / 100000$ in 1935 to $8 / 100000$ in $1983^{1}$ whereas the incidence of cardia carcinoma has increased at a rate of $5-10 \%$ per year over the last few decades. ${ }^{2}$ This increase in cardia carcinomas is comparable with the increase seen in distal oesophageal adenocarcinomas. ${ }^{23}$

According to the pathological tumour node metastasis criteria established by the Union Internationale Contre le Cancer (UICC) and the American Joint Committee on Cancer (AJCC), carcinoma of the gastric cardia is classified as gastric cancer, and carcinoma of the distal $8 \mathrm{~cm}$ of the oesophagus, including the intra-abdominal oesophagus, is classified as distal oesophageal cancer. ${ }^{4}{ }^{5}$ However, several studies suggest that this distinction in classification is rather artificial as a similar phenotype was found for these malignancies. ${ }^{6-9}$ Additionally, carcinomas originating from the gastric cardia (and invading the distal oesophagus) and those originating from the distal oesophagus are mostly treated as one clinical entity, by subtotal oesophagectomy and proximal gastrectomy, with no major differences in five year overall survival and survival according to tumour stage between the two groups. ${ }^{11}{ }^{11}$ Therefore, at present, a classification in which both carcinomas are regarded as one clinical entity is often favoured.

Apart from the common pattern of increasing incidence, a comparable prognosis, and the same optimal treatment options, it is questionable whether these two carcinomas have the same molecular characteristics and are one pathological entity. In a previous study, we demonstrated that the enzyme cyclooxygenase 2 (COX-2), which plays a central role in the production of prostaglandins, was upregulated in distal oesophageal adenocarcinomas developed in a Barrett segment. ${ }^{12}$ This COX-2 expression, which is associated with many oncogenetic processes in vitro (for example, decreased apoptosis, increased proliferation, angiogenesis, and invasiveness), ${ }_{13}^{13}$ was an independent prognostic factor for patients with a Barrett carcinoma. ${ }^{12}$ With the availability of selective COX-2 inhibitors, these findings justify the investigation of their role as a new chemopreventive or (neo-) adjuvant treatment strategy for this aggressive malignancy.

The aim of this study was to analyse expression of COX-2 in cardia carcinomas by using immunohistochemistry and to correlate COX-2 expression with clinicopathological parameters and survival in order to assess whether there might be a potential place for selective COX-2 inhibition in this malignancy. In addition, the results in cardia carcinomas were compared with those previously obtained by studying Barrett carcinomas to determine whether both upper gastrointestinal malignancies might have a comparable oncogenetic pathway.

Abbreviations: COX-2, cyclooxygenase 2 


\section{PATIENTS AND METHODS \\ Patients}

Between 1 January 1993 and 31 December 2000, 306 patients underwent oesophageal resection with proximal gastrectomy for adenocarcinoma of the oesophagus, gastro-oesophageal junction, or gastric cardia (invading the distal oesophagus) with curative intent (that is, locally resectable disease without distant metastases). Data from these 306 patients were prospectively collected in a database.

A total of 151 patients (of whom six were excluded during the immunohistochemical analyses) with a distal oesophageal adenocarcinoma developed in a histologically proven Barrett's oesophagus were analysed previously. ${ }^{12}$ The pathology reports of the remaining 155 patients were reviewed for the purpose of the present study. All patients were included who presented with an adenocarcinoma arising from the gastric cardia and substantially invading the distal oesophagus. The tumour was considered to be cardiac when no Barrett metaplasia was identified and when the epicentre was in the gastric cardia, defined as the area at and immediately below the gastro-oesophageal junction. ${ }^{15}$ Carcinomas with the epicentre of the mass located in the tubular oesophagus but without a Barrett segment were excluded, to prevent inclusion of cannibalised Barrett tumours $(n=15)$. Tumours arising from the fundus or the corpus of the stomach and infiltrating the gastric cardia or distal oesophagus were also excluded $(n=4)$, as were another two cases with an adenosquamous carcinoma. Thus 134 patients remained for further analysis.

For all patients, preoperative workup consisted of endoscopy with histological biopsy, external ultrasonography of the abdomen and neck, chest $x$ ray, endosonography, and indirect laryngoscopy. In 98 patients $(73.1 \%)$ resection was performed by a transhiatal approach without thoracotomy and extended lymph node dissection. Thirty six patients $(26.9 \%)$ underwent oesophagectomy through a right sided thoracotomy followed by a laparotomy in combination with two field lymph node dissection. Patients were followed until death or 1 July 2002, ensuring a minimal potential follow up of 18 months. Median actual follow up was 18 months (range 15 days to 7.8 years). They were seen on a regular basis for five years in the outpatient clinic. In the first two years, patients were seen at 3-4 month intervals, and afterwards at six month intervals. For the present study, patients and/or their family practitioners were contacted by phone to assess their current status when they had been discharged by the surgeon after five years. No patient was lost to follow up.

None of the patients received chemo- and/or radiotherapy preoperatively, and no adjuvant treatment was administered postoperatively. A limited number of patients received palliative external radiotherapy for symptomatic tumour recurrence. The study was done in accordance with the guidelines of the local ethics committee.

\section{COX-2 immunohistochemical staining}

The COX-2 immunohistochemical staining procedure is described in detail elsewhere. ${ }^{12}$ Briefly, formalin fixed paraffin embedded specimens were sectioned $(5 \mu \mathrm{m})$ and deparaffinised for antigen retrieval. Immunostaining was performed with a COX-2 specific mouse antihuman monoclonal antibody (160112; Cayman Chemical Co., Ann Arbor, Michigan, USA) at a dilution of 1:200. Every 20th sample of the trial series was a known colon adenocarcinoma specimen in which stromal cells at an area of ulceration were scored 3+, cancer cells from $2+$ to $3+$, and adjacent non-neoplastic epithelium $1+$ (for scoring criteria see below). Specificity of the antibody was confirmed by restaining a randomly selected subset of specimens (every loth sample, $\mathrm{n}=13$ ) with and without preadsorption of the primary antibody with a human COX-2 control peptide $(10 \mu \mathrm{g} / \mathrm{ml}$; Cayman Chemical) for one hour at room temperature prior to the staining procedure (that is, blocking controls).

COX-2 immunohistochemical staining was scored independently and in a blinded manner by two investigators (CB and AS). The following scoring criteria of tumour cells were agreed upon before the analysis: 0, no staining; $1+$, weak diffuse cytoplasmic staining (may contain stronger intensity in less than $10 \%$ of cancer cells); $2+$, moderate to strong granular cytoplasmic staining in 10-90\% of cancer cells; $3+$, over $90 \%$ of tumour cells stained with strong intensity. Scores 0 and 1 were categorised as "COX-2 low" and scores 2 and 3 as "COX-2 high" for the statistical analyses (see below). Allocation of tumours to the "COX-2 low" versus the "COX-2 high" category by the two investigators was similar ( $>90 \%$ of specimens were categorised identically). In cases of disagreement $(n=14)$ the slides were re-evaluated using a multiheaded microscope (CB, AS, and AR). These scoring criteria and previously described immunohistochemical control procedures are identical to those used in our previous report on COX-2 expression in oesophageal adenocarcinoma. ${ }^{12}$

\section{Statistical analysis}

The association between demographic and clinicopathological features and COX-2 expression was analysed using the Student's $t$ test (continuous data) and the $\chi^{2}$ test (categorical data). Overall survival was estimated according to the Kaplan-Meier method and compared using the log rank test. The Cox proportional hazard model was used to evaluate various factors simultaneously.

A p value of $\leqslant 0.05$ was considered statistically significant. All statistical analyses were performed using the Statistical Software Package version 9.0 (SPSS Inc., Chicago, Illinois, USA).

\section{RESULTS}

\section{Patients}

There were 113 males (84.3\%) and 21 females (15.7\%), with a median age of 64 years (range 39-83).

\section{Expression of COX-2 protein in adenocarcinomas of the gastric cardia}

COX-2 immunoreactivity was detected in 112 of 134 (86.7\%) adenocarcinomas of the cardia whereas 22 tumours had no COX-2 expression. Moderate to strong staining ("COX-2 high") with a granular cytoplasmic pattern was observed in $55 / 134(41.0 \%)$ cases of which two were scored as strong and 53 as moderate. COX-2 expression was mainly localised in the neoplastic cells of the invading peripheral margin of the tumour. Superficial tumour cells towards the lumen were often less intense, and only weak or no staining was observed in stromal cells (connective tissue cells, smooth muscle cells, and blood vessels), except at sites of erosions and ulcerations (fig 1).

\section{Correlation between COX-2 expression and pathological or clinical parameters}

COX-2 expression was not significantly correlated with any clinicopathological parameter at the time of operation, although a possible trend was seen towards a positive association with the presence of lymph node metastases $(p=0.08)$ (table 1$)$. Neither could any correlation be found between elevated COX-2 expression and the development of distant metastases or locoregional recurrences during follow up (table 1).

Kaplan-Meier curves for patient survival are depicted in fig $2 \mathrm{~A}$. As can be seen in the survival curves, there was no significant difference in survival between patients in the 


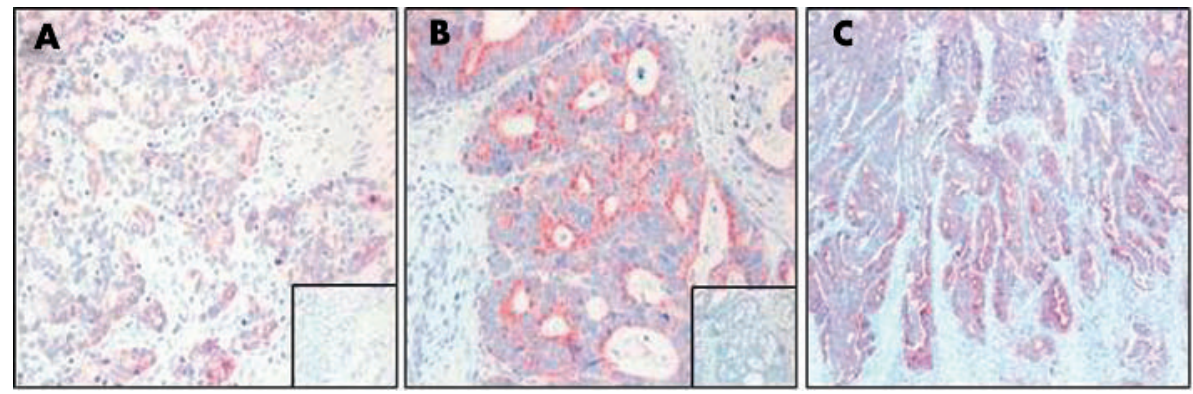

Figure 1 Representative examples of cyclooxygenase 2 (COX-2) immunohistochemistry. (A) Weak (1+) immunoreactivity in tumour cells. This tumour was categorised as "COX-2 low" (200x). In the lower right corner the blocking control is shown (see materials and methods for details). (B) Moderate $(2+)$ immunoreactivity in tumour cells. This tumour was categorised as "COX-2 high" $(200 \times)$. In the lower right corner the blocking control is shown. (C) Immunoreactivity was stronger in the invading peripheral margin of the tumour.

\begin{tabular}{|c|c|c|c|}
\hline & \multicolumn{2}{|c|}{ COX-2 expression } & \multirow[b]{2}{*}{ p Value } \\
\hline & $\begin{array}{l}\text { Low }(n=79) \\
(n(\%))\end{array}$ & $\begin{array}{l}\text { High }(n=55) \\
(n(\%))\end{array}$ & \\
\hline \multicolumn{4}{|l|}{ Patient characteristics } \\
\hline Age (mean (SD)) (y) & $64(10)$ & $62(9)$ & 0.3 \\
\hline \multicolumn{4}{|l|}{ Sex } \\
\hline Male & 70 (88.6) & 43 (78.2) & \multirow[t]{2}{*}{0.1} \\
\hline Female & $9(11.4)$ & $12(21.8)$ & \\
\hline \multicolumn{4}{|l|}{ Tumour characteristics } \\
\hline Depth of invasion* & & & \\
\hline $\mathrm{T1}$ & $4(5.1)$ & $1(1.8)$ & \multirow[t]{4}{*}{0.7} \\
\hline T2 & $6(7.6)$ & $3(5.5)$ & \\
\hline T3 & $64(81.0)$ & $48(87.3)$ & \\
\hline $\mathrm{T} 4$ & $5(6.3)$ & $3(5.5)$ & \\
\hline \multicolumn{4}{|c|}{ Lymph node involvementt } \\
\hline NO & $17(21.5)$ & $9(16.4)$ & \multirow[t]{4}{*}{0.08} \\
\hline $\mathrm{N} 1$ & $37(46.8)$ & $27(49.1)$ & \\
\hline N2 & $24(30.4)$ & $13(23.6)$ & \\
\hline N3 & $1(1.3)$ & $6(10.9)$ & \\
\hline \multicolumn{4}{|l|}{ Distant metastasis } \\
\hline MO & $75(94.9)$ & $53(96.4)$ & \multirow[t]{2}{*}{0.5} \\
\hline MI & $4(5.1)$ & $2(3.6)$ & \\
\hline \multicolumn{4}{|l|}{ Differentiation grade } \\
\hline Well & $2(2.5)$ & $1(1.8)$ & \multirow[t]{3}{*}{0.7} \\
\hline Moderate & $29(36.7)$ & $17(30.9)$ & \\
\hline Poor & $48(56.4)$ & $37(67.3)$ & \\
\hline \multicolumn{4}{|l|}{ Tumour stageł } \\
\hline la & $3(3.4)$ & $0(0.0)$ & \multirow[t]{6}{*}{0.7} \\
\hline $\mathrm{lb}$ & $1(1.3)$ & $2(3.6)$ & \\
\hline ॥ & $16(20.3)$ & $10(18.2)$ & \\
\hline Illa & $32(40.5)$ & $25(45.5)$ & \\
\hline IIlb & $23(29.1)$ & $16(29.1)$ & \\
\hline IV & $4(5.1)$ & $2(3.6)$ & \\
\hline \multicolumn{4}{|l|}{ Operation type§ } \\
\hline THE & $58(73.4)$ & 40 (72.7) & \multirow[t]{2}{*}{0.5} \\
\hline TE & $21(26.6)$ & $15(27.3)$ & \\
\hline \multicolumn{4}{|l|}{ Radicality of resection } \\
\hline RO & 58 (73.4) & $40(72.7)$ & \multirow[t]{3}{*}{0.5} \\
\hline R1 & $21(26.6)$ & $14(25.5)$ & \\
\hline R2 & $0(0.0)$ & $1(1.8)$ & \\
\hline \multicolumn{4}{|c|}{ Clinical outcome } \\
\hline \multicolumn{4}{|c|}{ Locoregional recurrence } \\
\hline No & $41(51.9)$ & $31(56.4)$ & \multirow[t]{2}{*}{0.6} \\
\hline Yes & $38(48.1)$ & $24(43.6)$ & \\
\hline \multicolumn{4}{|l|}{ Distant metastasis } \\
\hline No & $45(57.0)$ & $31(56.4)$ & \multirow[t]{2}{*}{0.9} \\
\hline Yes & $34(43.0)$ & $24(43.6)$ & \\
\hline
\end{tabular}

${ }^{*} \mathrm{~T} 1$, tumour limited to the submucosa; $\mathrm{T} 2$, tumour infiltrates muscularis propria, but not adventitia; $\mathrm{T} 3$, tumour infiltrates adventitia; $\mathrm{T} 4$, tumour infiltrates adjacent structures.

†NO, no lymph node metastasis; N1, 1-6 lymph node metastases; N2, 7-15 lymph node metastases; N3, more than 15 lymph node metastases.

fla, T1NOMO; Ib, TIN1MO, T2NOMO; II, TIN2-3MO, T2N1MO, T3NOMO; Illa, T2N2-3MO, T3N1MO; Illb, T3N2$3 M 0$, TAN1MO; IV, T4N2-3MO, anyTanyNM1. §THE, transhiatal resection; TTE, transthoracic resection. $\bullet \mathrm{RO}$, microscopically radical; R1, microscopically non-radical; R2, macroscopically non-radical. 
"COX-2 low" category compared with the "COX-2 high" category, with a median survival of 22 months (95\% confidence interval (CI) 13-31) and 25 months (95\% CI $8-42)$, respectively $(p=0.50 ; \log$ rank test). Subgroup analysis of the prognostic value of COX-2 per tumour stage did not show any significant difference in survival. Because this might be due to the low numbers of patients available per tumour stage, tumour stage was recoded into a dichotomous variable by combining categories with comparable prognosis (tumour stages Ia, Ib, II $v$ IIIa, IIIb, IV). There was also no significant survival advantage for patients with "COX-2 low" expression in either group, although there was a slight indication that elevated COX-2 expression was a predictor for poor outcome in relatively early cancers $(\mathrm{p}=0.17)($ fig $2 \mathrm{~B})$.

\section{Comparison with oesophageal Barrett carcinoma}

The finding that COX-2 expression was not a prognostic variable for cardia carcinomas is different from results of distal oesophageal adenocarcinomas in which it was shown to be an independent prognostic variable together with tumour stage and radicality of resection. ${ }^{12}$ Comparison of the COX-2 staining results showed significantly less intense COX-2 staining in cardia carcinomas compared with Barrett carcinomas $(\mathrm{p}=0.0001)$ (table 2$)$.

Overall, there was no significant difference in survival between patients with a gastric cardia carcinoma and patients with a Barrett carcinoma $(\mathrm{p}=0.22$; log rank test), although a trend was seen towards a more favourable outcome for patients with a Barrett carcinoma (median 25 months (95\% CI 18-33) v median 38 months (95\% CI 16-60), respectively). This can be explained, at least in part, by a more favourable $\mathrm{T}$ and $\mathrm{N}$ stage in patients with a Barrett carcinoma $(\mathrm{p}<0.001)$.

\section{DISCUSSION}

This study shows that elevated COX-2 expression is present in $41 \%$ of patients (moderate to strong) with a cardia carcinoma. However, it did not correlate with prognosis. This finding is in contrast with our previous study in which we demonstrated that elevated COX-2 expression was an independent prognostic variable for Barrett carcinomas, ${ }^{12}$ and is surprising in view of the epidemiological and clinical similarities (that is, rapidly rising incidence, stage by stage prognosis, and optimal surgical treatment) shared by both distal oesophageal and gastric cardia adenocarcinomas. ${ }^{6-8} 16$ However, it is consistent with the results of a large epidemiological study in which a substantial protective effect of non-steroidal anti-inflammatory drugs was observed for adenocarcinomas arising at distal gastric or oesophageal sites but not for cardia caricnomas. ${ }^{17}$

A possible explanation for the discrepancy in prognostic relevance of COX-2 that we identified between adenocarcinomas of the gastric cardia and distal oesophagus may be a difference in carcinogenesis for these two cancers. Apart from the significant difference in the intensity of COX-2 expression in carcinoma cells, there was a discrepancy in the pattern of expression. In cardia carcinomas, the strongest (heterogeneous) COX-2 expression was seen in the invading peripheral margin of the tumour, in contrast with the homogeneous and predominantly superficial luminal expression of COX-2 seen in Barrett carcinoma. This suggests that in cardia carcinomas, COX-2 upregulation is a relatively late event and occurs in invasive malignant cells that are often already infiltrating the lymphatic system or blood vessels, which could explain why COX-2 expression is not a prognostic factor.

This difference in COX-2 expression with respect to intensity, localisation, and prognostic significance is suggestive of a different pathogenesis and different genetic constitution of these two adenocarcinomas. To date, the strongest supportive evidence that Barrett and cardia carcinomas represent two separate clinical entities has come from an epidemiological study identifying symptomatic reflux as a strong risk factor for oesophageal adenocarcinomas, and only a relatively weak risk factor for adenocarcinomas of the gastric cardia. ${ }^{18}$ Other evidence indicating that these tumours should be regarded as two different clinical entities comes from recent reports comparing intestinal metaplasia of the gastric cardia with that of the distal oesophagus. ${ }^{19} 20$ Intestinal metaplasia of the cardia is predominantly of the complete type and is associated with pathological features of the stomach, especially pangastritis. This is in contrast with the incomplete type of intestinal metaplasia in the distal oesophagus (that is, Barrett mucosa), which is characterised by the presence of goblet cells and non-secretory columnar cells and carries an increased risk of
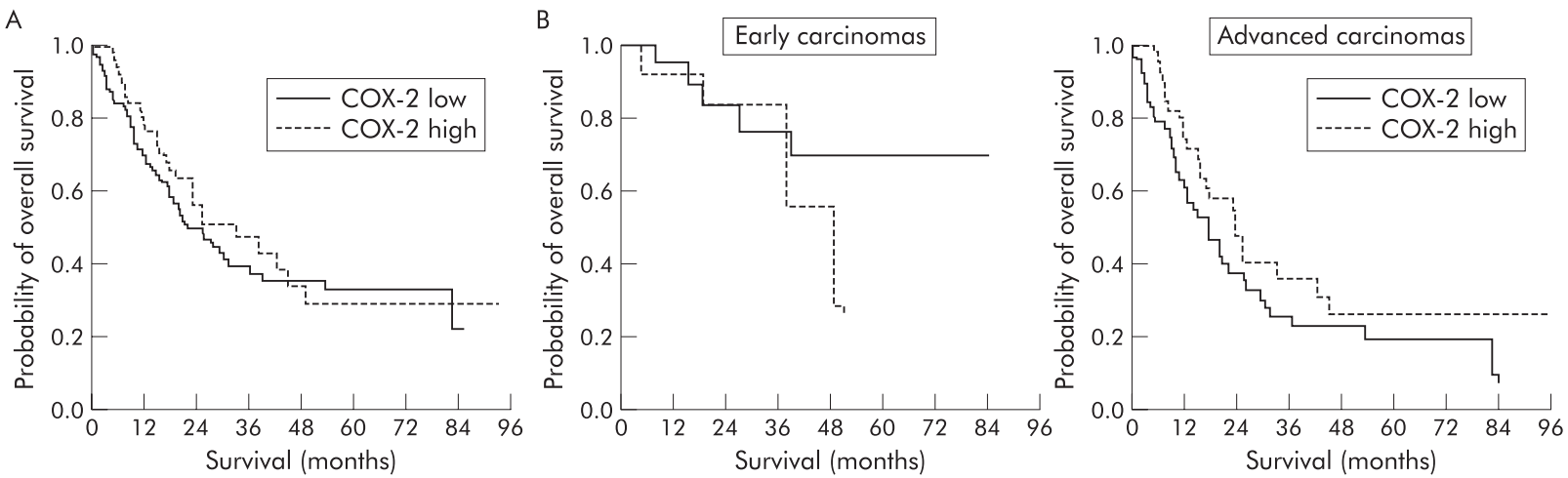

\begin{tabular}{|l|c|c|c|c|c|c|c|c|}
\hline & \multicolumn{10}{|c|}{ Patients at risk } \\
\hline COX-2 low & 79 & 49 & 28 & 21 & 17 & 9 & 5 & 1 \\
\hline COX-2 high & 55 & 40 & 22 & 11 & 7 & 3 & 2 & 1 \\
\hline
\end{tabular}

Figure 2 (A) Kaplan-Meier curves for 134 patients with an adenocarcinoma of the gastric cardia. There were 79 patients with low expression of cyclooxygenase 2 ("COX-2 low") and 55 with "COX-2 high" expression. No significant difference was observed between the two groups ( $p=0.50$; log rank test). (B) Kaplan-Meier curves of 32 patients with relatively early adenocarcinoma of the gastric cardia (stages la, Ib, and II) versus 102 patients with a more advanced tumour stage (stages IIla, IIIb, and IV). There was no significant difference in survival between patients in the "COX-2 low" category compared with the "COX-2 high" category, but there was a trend towards elevated COX-2 expression being a predictor of reduced survival in early carcinomas compared with more advanced tumours ( $p=0.17 v p=0.41$, respectively). 
Table 2 Comparison of cyclooxygenase 2 (COX-2) staining between adenocarcinomas of the gastric cardia (present study) and adenocarcinomas of the distal oesophagus developed in a Barrett segment (Buskens and colleagues ${ }^{12}$ )

\begin{tabular}{|c|c|c|c|}
\hline \multirow[b]{2}{*}{ COX-2 expression } & \multicolumn{3}{|l|}{ Tumour localisation } \\
\hline & Cardia $(n=134)(n(\%))$ & Oesophagus $(n=145)(n(\%))$ & p Value \\
\hline $\begin{array}{l}0(24) \\
1(85) \\
2(155) \\
3(15)\end{array}$ & $\begin{array}{c}22(16.4) \\
57(42.5) \\
53(39.6) \\
2(1.5)\end{array}$ & $\begin{aligned} 2 & (1.4) \\
28 & (19.3) \\
102 & (70.3) \\
13 & (9.0)\end{aligned}$ & 0.0001 \\
\hline
\end{tabular}

In cardia carcinomas, significantly less (intense) COX-2 staining was found ( $p=0.0001$ ).

dysplasia or cancer. ${ }^{20}$ Adenocarcinomas of the cardia were also reported to have a different oncogenetic profile compared with distal oesophageal carcinomas. ${ }^{21} 22$ In particular, the prevalence of $\mathrm{p} 53$ mutations in cardia carcinomas is less than $50 \%(31-42 \%)^{21} 23$ whereas for oesophageal carcinomas a p53 mutation is the most frequent alteration identified (75-100\%). ${ }^{24}{ }^{25}$ As p53 mutations induce COX-2 transcription in vitro by disrupting binding of p53 to the promoter region of COX-2, this could be an explanation for the less pronounced COX-2 upregulation in cardia carcinomas in comparison with Barrett carcinomas. ${ }^{25} 26$

Another explanation for the difference in prognostic value of COX-2 for adenocarcinomas of the cardia and adenocarcinomas of the distal oesophagus arising in a Barrett segment might be that elevated expression of COX-2 is only a prognostic variable for poor prognosis in relatively early carcinomas. In lung cancer, upregulation of COX-2 was demonstrated to be associated with worse overall survival in patients with stage I non-small cell lung cancer. ${ }^{27}{ }^{28}$ This would be in line with the prognostic significance of COX-2 expression for patients with Barrett carcinomas who had significantly more favourable $\mathrm{T}$ and $\mathrm{N}$ stages than patients with cardia carcinomas. This is also supported by the finding that there seemed to be a trend for a prognostic role for COX2 in early carcinomas of the gastric cardia compared with more advanced tumour stages.

In this report, we analysed the clinical significance of elevated COX-2 expression in adenocarcinomas of the gastric cardia. We could not demonstrate that COX-2 expression in epithelial tumour cells was related to stage or poorer long term outcome for patients with adenocarcinoma of the gastric cardia, which is in contrast with our previous results in Barrett carcinomas where COX-2 expression was an independent prognostic variable for patient survival. ${ }^{12}$ These results support the hypothesis that although these cancers might be considered as one clinical entity, they are two distinct pathological entities.

There are several ongoing chemoprevention and adjuvant chemotherapy trials with selective COX-2 inhibitors for gastrointestinal malignancies. ${ }^{29}$ Although the role of selective COX-2 inhibitors in the prevention and treatment of gastrointestinal cancer is promising, ${ }^{30}$ further investigations are needed before they can be incorporated into daily clinical practice. This study demonstrates that different tumours may require different treatment strategies. Therefore, it is important to gain further insight in the mechanisms by which COX-2 contributes to the carcinogenesis of various cancers. To date, these findings make the role of selective COX-2 inhibitors in the treatment of adenocarcinomas of the gastric cardia less promising than in Barrett cancers.

\section{ACKNOWLEDGEMENTS}

The authors greatly acknowledge Eric Caspers, Folkert Morsink, and Alex Musler from the AMC, Department of Pathology, and Elina Laitinen from Helsinki University Central Hospital, for excellent technical assistance. CJ Buskens and BP van Rees were supported by travel grants from the Netherlands Organisation for Scientific Research (NWO-MW) and the Academy of Finland. A Sivula, C Haglund, and A Ristimäki were supported by the Academy of Finland, the Finnish Cancer Foundation, Finska Läkaresällskapet, and Helsinki University Central Hospital Research Funds.

\section{Authors' affiliations}

C J Buskens, J J B van Lanschot, Department of Surgery, Academic Medical Centre/University of Amsterdam, Amsterdam, the Netherlands A Sivula, A Ristimäki, Department of Pathology, Helsinki University Central Hospital and Molecular and Cancer Biology Research Program, Biomedicum Helsinki, University of Helsinki, Helsinki, Finland

B P van Rees, G J A Offerhaus, Department of Pathology, Academic Medical Centre/University of Amsterdam, Amsterdam, the Netherlands C Haglund, Department of Surgery, Helsinki University Central Hospital, University of Helsinki, Helsinki, Finland

\section{REFERENCES}

1 Wanebo HJ, Kennedy BJ, Chmiel J, et al. Cancer of the stomach. A patient care study by the American College of Surgeons. Ann Surg 1993;218:579-82.

2 Blot WJ, Devesa SS, Kneller RW, et al. Rising incidence of adenocarcinoma of the esophagus and gastric cardia. JAMA 1991;265:1287-9.

3 Powell J, McConkey CC. The rising trend in oesophageal adenocarcinoma and gastric cardia. Eur $J$ Cancer Prev 1992;1:265-9.

4 Sobin LH, Wittekind C, eds. International Union Against Cancer TNM classification of malignant tumours, 6th edn. New York: John Wlley and Sons, 2002

5 American Joint Committee on Cancer. AJCC cancer staging manual, 5th edn. Chigago: American Joint Committee on Cancer, 1997.

6 Chow WH, Blot WJ, Vaughan TL, et al. Body mass index and risk of adenocarcinomas of the esophagus and gastric cardia. J Natl Cancer Inst 1998;90:150-5.

7 Gammon MD, Schoenberg JB, Ahsan H, et al. Tobacco, alcohol, and socioeconomic status and adenocarcinoma of the esophagus and gastric cardia. J Natl Cancer Inst 1997:89:1277-84.

8 MacDonald WC, MacDonald JB. Adenocarcinoma of the esophagus and/or gastric cardia. Cancer 1987;60:1094-8.

9 Mendes de Almeida JC, Chaves P, Pereira AD, et al. Is Barrett's esophagus the precursor of most adenocarcinomas of the esophagus and cardia? A biochemical study. Ann Surg 1997;226:725-33.

10 Wijnhoven BL, Siersema PD, Hop WCJ, et al. Adenocarcinomas of the distal oesophagus and gastric cardia are one clinical entity. Br J Surg 1999:86:529-35.

11 Steup WH, De Leyn P, Deneffe G, et al. Tumors of the esophagogastric junction. Long-term survival in relation to the pattern of lymph node metastasis and a critical analysis of the accuracy or inaccuracy of pTNM classification. J Thorac Cardiovasc Surg 1996;111:85-94.

12 Buskens CJ, van Rees BP, Sivula A, et al. Prognostic significance of elevated cyclooxygenase-2 expression in patients with adenocarcinoma of the esophagus. Gastroenterology 2002;122:1800-7.

13 Dannenberg AJ, Altorki NK, Boyle JO, et al. Cyclo-oxygenase 2: a pharmacological target for the prevention of cancer. Lancet Oncol $2001 ; 2: 544-51$.

14 Gupta RA, Dubois RN. Colorectal cancer prevention and treatment by inhibition of cyclooxygenase-2. Nat Rev Cancer 2001;1:11-21.

15 Stein HJ, Feith M, Siewert JR. Cancer of the esophagogastric junction. Surg Oncol 2000;9:35-41.

16 Pera M. Epidemiology of esophageal cancer, especially adenocarcinoma of the esophagus and esophagogastric junction. Recent Results Cancer Res 2000;155:1-14.

17 Farrow DC, Vaughan TL, Hansten PD, et al. Use of aspirin and other nonsteroidal anti-inflammatory drugs and risk of esophageal and gastric cancer. Cancer Epidemiol Biomarkers Prev 1998;7:97-102. 
18 Lagergren J, Bergstrom R, Lindgren A, et al. Symptomatic gastroesophageal reflux as a risk factor for esophageal adenocarcinoma. N Engl J Med 1999;340:825-31.

19 Balaji NS, DeMeester SR, Wickramasinghe KS, et al. Etiology of intestinal metaplasia at the gastroesophageal junction. Surg Endosc 2002;10:1403-8.

20 Van Sandick JW, Van Lanschot JJB, Van Felius L, et al. Intestinal metaplasia of the esophagus or esophagogastric junction: evidence of distict clinical, pathologic, and histochemical staining features. Am J Clin Pathol 2002; 117:117-25.

21 Taniere P, Martel-Planche G, Maurici D, et al. Molecular and clinica differences between adenocarcinomas of the esophagus and of the gastric cardia. Am J Pathol 2001;158:33-40.

22 Van Dekken H, Geelen E, Dinjens WNM, et al. Comparative genomic hybridization of cancer of the gastroesophageal junction: Deletion of 14Q3132.1 discriminates between esophageal (Barrett's) and gastric cardia adenocarcinomas. Cancer Res 1999;59:748-52.

23 Flejou JF, Gratio V, Muzeau F, et al. p53 abnormalities in adenocarcinoma of the gastric cardia and antrum. Mol Pathol 1999;52:263-8.
24 Krishnadath KK, Tilanus HW, van Blankenstein M, et al. Accumulation of p53 protein in normal, dysplastic, and neoplastic Barrett's oesophagus. J Pathol 1995; 175:175-80

25 Neshat K, Sanchez CA, Galipeau PC, et al. p53 mutations in Barrett's adenocarcinoma and high-grade dysplasia. Gastroenterology 1994; 106:1589-95.

26 Subbaramaiah K, Altorki N, Chung WJ, et al. Inhibition of cyclooxygenase-2 gene expression by p53. J Biol Chem 1999;274:10911-5.

27 Khuri FR, Wu H, Lee JJ, et al. Cyclooxygenase- 2 overexpression is a marker of poor prognosis in stage I non-small cell lung cancer. Clin Cancer Res 2001;7:861-7.

28 Brabender JP, Park J, Metzger R, et al. Prognostic significance of cycloxygenase- 2 mRNA expression in non-small cell lung cancer. Ann Surg 2002;235:896-7.

29 Chau I, Cunningham D. Cyclooxygenase inhibition in cancer-a blind alley or a new therapeutic reality? N Engl J Med 2002;346:1085-7.

30 Whittle BJR. COX-1 and COX-2 products in the gut: therapeutic impact of COX-2 inhibitors. Gut 2000;47:320-5. 